\title{
Sonorité, Espace et Forme Dans \\ "La Cathédrale engloutie" de Debussy
}

\author{
Didier Guigue
}

\section{Introduction}

«Remuement informe des profondeurs», 《résonances archaïques» symbolisant «les forces mystiques celtes» et «le rite païen de la mer» 1 , La Cathédrale engloutie semble exemplairement l'oeuvre au sujet de laquelle le discours analytique ne tend que trop souvent à se retrancher derrière une profusion de métaphores poétiques de peu de recours pour sa compréhension formelle. Or, Jarocinsky a bien insisté sur le fait que tout examen « qui n'aborde pas les valeurs purement sonores qu'elle recèle, doit être jugé incomplet, partiel et déformant son véritable aspect » 2 . Toutefois, la plupart des travaux qui, cherchant à analyser la configuration et le rôle formel des sonorités dans ce Prélude, vont au-delà de la description métaphorique, abordent cet aspect par des moyens qui ne permettent que des observations très générales 3 , ou la description locale d'effets pianistiques de timbre, sans pouvoir préciser techniquement leurs dynamiques interne et externe 4.

Le présent travail cherche à pallier partiellement à ce déficit, en proposant une méthode spécifique d'investigation de la partition, qui soit à même de fournir des réponses strictement techniques sur les stratégies mises en oeuvre par le compositeur pour articuler un des composants de l'écriture qui participe de la configuration de ces « valeurs purement sonores » dont parle Jarocinsky, à savoir : l'utilisation de l'espace5. 
Par espace, nous entendons spécifiquement ici les modalités d'investissement de l'étendue du piano, indépendamment des lois d'organisation verticale des hauteurs. Ces modalités reflètent les choix du compositeur en regard de la dimension proprement sonore qu'il entend attribuer à chaque instant de l'oeuvre. Plus l'étendue investie est grande, plus la qualité sonore de l'objet musical qu'elle instantie devient riche et complexe. Cette complexité s'accroîtra encore proportionnellement au nombre de sons qui l'occupent et à leur distribution à l'intérieur de cet espace. On appellera, dans la suite de cette discussion, qualité sonore ce taux et ces modalités d'occupation de l'étendue complète de l'instrument, tout en gardant bien à l'esprit que la gestion de l'espace n'est assurément pas le seul moyen permettant de moduler cette qualité

Pour ne pas partir d'un niveau zéro d'analyse, nous demanderons au lecteur d'accepter par hypothèse le découpage formel du Prélude tel que l'a exposé Roy Howat dans son ouvrage sur les proportions dans l'oeuvre de Debussy 6. Cet auteur décrit la structure séquentielle générale de La Cathédrale engloutie par la métaphore architecturale de la voûte, c'est-à-dire par une structure présentant une dynamique symétrique de part et d'autre d'un "faîte" - une forme qu'il schématise par la séquence alphabétique $\mathrm{ABCBA}$. Cette proposition semble à priori assez convaincante, même si l'on ne suit pas l'auteur dans ses thèses sur les pratiques occultes et numérologiques de Debussy 7 . En adoptant ce schéma formel, la "coda" finale (mm.84-89) n'est autre que le versant symétrique de l' "introduction" (mm. 1-27): ce sont les deux versants de la section $\mathrm{A}$, qui se caractérisent par la présence presque exclusive du seul motif de base de l'oeuvre8, que nous étiquetterons dorénavant $\mathrm{M}$. La section B incarne le "thème en Do" dans ses deux présentations en miroir (mm.28-46 et 72-83)9. Et C, section "dominante" (mm.47-67)10, construite à partir du "deuxième thème" 11 , amène, "dans une expression allant grandissant" jusqu'à l'épicentre (mm.59-62), le climax de la pièce.

Il est intéressant de voir comment les paratextes du compositeur en tête de chaque section sont parfaitement cohérents avec cette dynamique formelle : 

$\mathrm{A}=$ "Dans une brume doucement sonore"
$\mathrm{B}=$ "Sonore sans dureté"
$\mathrm{C}=$ "Dans une expression allant grandissant"
$\mathrm{B}=$ "Comme un écho de la phrase entendue précédemment" (i.e. le premier versant de B)
$\mathrm{A}=$ "Dans la sonorité du début".

Cet itinéraire paratextuel est le meilleur indice en faveur de la proposition de Howat. Mais dans ces conditions il manque à cette architecture une section que le compositeur a paratexté "peu à peu sortant de la brume" ( $\mathrm{mm} .16-27)$, et qui par cette indication semble clairement être conçue comme un agent de liaison/transition entre A et $\mathrm{B}$, ou, plus globalement, comme un palier supplémentaire dans la progression ascendante qui conduit de A à C. C'est probablement tenu par la rigueur, pourtant assouplie, des contraintes imposées par le paradigme de la divine proportion - la section d'or - sur lequel il fonde son approche formelle de Debussy, que l'auteur a préféré occulter cette section dans son découpage. N'ayant pas signé le même contrat, nous nous sommes permis d'incorporer au schéma de Howat une subdivision supplémentaire entre $A$ et $B$.

D'autre part, nous avons choisi de substituer à l'étiquetage alphabétique un étiquetage numérique dont les valeurs correspondraient à la "position" des sections sur un arc de cercle de base (0) et de sommet (1), conformément au modèle de la voûte. Ainsi, la base (0) est le lieu des sections liminaires A, et le sommet (1), celui de la section culminante $\mathrm{C}$. En conséquence, les sections médianes (B) se voient attribuer la position médiane $(0.5)$, et proportionnellement, la section transitoire entre $\mathrm{A}$ et $\mathrm{B}$, non reconnue par Howat, la position (0.25). Cette numérisation a pour seul but de permettre le calcul des corrélations entre la structure formelle et les évaluations numériquement quantifiées de l'utilisation de l'espace. Pour différencier deux sections de même valeur numérique (c'est-à-dire les deux versants d'une même entité), celleci sera affectée à sa droite du signe + pour les sections situées sur le versant ascendant de l'arc de cercle, et du signe - pour celles situées sur le versant descendant. Ainsi la séquence formelle se décrit-elle : $\quad(\mathrm{S} 0+, \mathrm{S} 0.25, \mathrm{~S} 0.5+, \mathrm{S} 1, \mathrm{~S} 0.5-, \mathrm{S} 0-) 12$. 


\section{2. "Thème-sujet" et "thème-objet"}

À juste titre, la littérature de référence a relevé la forte singularité de la section dite "en Do", énoncée une première fois en 28 puis reprise "comme un écho" en 72. Son statisme et son invariance sous nombre de dimensions - mais pas toutes - pourrait typiquement correspondre à ce que Barraqué a appelé un "thème-objet", «dont la fonction essentielle [...] est d'assurer, à certains moments de clivage, un rôle proprement passif $\gg 13$. Nous n'irons point jusqu'à tenir que, dans le cas de ce prélude, ce "thème" est passif, puisqu'il incorpore des éléments motivico-thématiques essentiels, et qu'il participe très activement de la structure formelle de l'ensemble. Nous conserverons toutefois ici du concept de "thème-objet" l'idée d'invariance thématico-sonore, qui implique un trait stylistique très debussyen, à savoir l'absence de développement. Cet objet, dans sa deuxième itération, pourrait cristalliser cette expression suprême de la désagrégation qu'a vu Jankélévitch dans cette oeuvre : il serait « le reflet d'une chose enfouie dans les profondeurs [...], une architecture tremblante et diffluente ».

Par analogie, nous qualifierons le "deuxième thème" dit en "Do\#" ( $\mathrm{mm} .7-13$ et 47-67) en fonction de son comportement et de sa structure. En effet, alors que le "thème-objet" (dorénavant TO) est statique, le "thème en Do\#" est dynamique dans la mesure où il génère un véritable développement dans la section $\mathrm{C}$, et où le matériau harmonique est fluctuant, moins stabilisé que celui de TO14. Ces caractéristiques dynamiques, parmi d'autres qui pourraient être révélées à une analyse plus minutieuse (fluctuations des registres et des intensités en particulier), inclinent à situer ce thème en opposition dialectique au $\mathrm{TO}$, d'une manière analogue, toutes précautions prises, aux formes sonate bithématiques 15 . C'est pourquoi nous l'étiquetterons dorénavant "thème-sujet" (TS), sans que ceci implique une quelconque prédominance hiérarchique. Ici, "sujet" et "objet" sont des attributs du comportement formelfonctionnel de ces thèmes, dans un sens barraquéen d'activité (dynamisme, variance) ou de passivité (statisme, invariance). Voici donc sur quel schéma macro-formel nous allons aborder notre analyse de l'organisation de l'espace dans La Cathédrale engloutie: (Fig. 1). 


\begin{tabular}{|c|c|c|c|c|c|}
\hline HOWAT & GUIGUE & mes & $\begin{array}{l}\text { CONIENU } \\
\text { MOTIVICO- } \\
\text { THEMATIQUE }\end{array}$ & $\begin{array}{l}\text { REGION POLE } \\
\text { HARMONIQUE }\end{array}$ & PARATEXTE \\
\hline \multirow[t]{2}{*}{ A } & $\mathrm{SO}^{+}$ & 1 & $M+T S$ & $\begin{array}{l}\text { SOL MI } \\
(\text { do } \#) \text { DO }\end{array}$ & $\begin{array}{c}\text { Dans une brume } \\
\text { doucement } \\
\text { sonore }\end{array}$ \\
\hline & So.25 & 16 & M & $\begin{array}{l}\text { SI MIb V de } \\
\text { DO }\end{array}$ & $\begin{array}{c}\text { Peu à peu sortant de la } \\
\text { brume }\end{array}$ \\
\hline B & So. $5+$ & 28 & TO & DO & Sonore sans dureté \\
\hline $\mathrm{C}$ & $\mathrm{S} 1$ & 47 & TS & $\mathrm{SOL} \#($ do $\#)$ & $\begin{array}{c}\text { Dans une expr. allant } \\
\text { grandissant }\end{array}$ \\
\hline B & So.5- & 72 & TO & DO & Comme un ècho $[\ldots]$ \\
\hline$A$ & SO- & 84 & M & DO & $\begin{array}{c}\text { Dans la sonorité du } \\
\text { début }\end{array}$ \\
\hline
\end{tabular}




\subsection{Segmentation du texte musical}

La saisie d'un texte musical pour l évaluation des composants de 1 écriture susceptibles de contrôler l espace, passe par sa segmentation en une liste discrète de groupes globalement homogènes, segmentation qui ne saurait valablement se contenter de manière systématique de celle que forme la séquence des mesures 16. Nous proposons d'établir une méthode de segmentation basée sur la notion de rupture de continuité dans un ou plusieurs composants de l écriture, rupture qui signale le passage discret d'un objet homogène à 1 autre.

Nous justifions et décrivons ailleurs en détail la procédure de segmentation adoptée pour cette lecture 17. Disons ici succinctement qu'elle implique les ruptures au niveau de la macroforme (silences, points d'orgues...), les interruptions de pédales et/ ou de liaisons d'articulation, ainsi que les ruptures de continuité dans les intensités, les registres, les configurations rythmiques, les densités, etc... Ces critères se veulent aveugles en regard des principes d'organisation périodique et/ou mélodico-harmonicothématique adoptés par le compositeur, ce qui n'empêche pas leur éventuelle coïncidence, surtout à un niveau hiérarchique élevé. Cette indépendance est fondamentale pour pouvoir évaluer les rapports que 1 ensemble des dimensions du langage musical peut entretenir avec cette écriture des sonorités.

En application de cette méthode, La Cathédrale engloutie a été segmentée en 43 "objets" étiquetés $\mathrm{O}$ et numérotés séquentiellement de 1 à 43 (cf. partition annexée). Puis, chacun d'entre eux a fait 1 objet d'une évaluation de ses caractéristiques d'occupation de 1 espace. Ces évaluations sont assistées par des algorithmes informatiques que nous avons implémentés dans l environnement logiciel Patchwork développé à I'IRCAM.

\section{3. Évaluation des attributs de l'écriture de l'espace}

\subsection{L'écriture de l'espace au piano}

L'analyse de l'écriture de l'espace dans une oeuvre pour piano consiste à interpréter, c'est-à-dire évaluer, la partition - plus 
précisément les unités en lesquelles elle aura été préalablement segmentée -, sur les composants dont on aura déterminé qu'ils apportent les informations nécessaires et suffisantes pour décrire la façon dont le compositeur gère l'espace des hauteurs qu'il a à sa disposition. En l'occurrence, ces évaluations concernent la nature et le comportement de deux composants de l'écriture, à savoir l'ambitus relatif (dorénavant AMBITUS), et la registration (dorénavant REGISTRE). AMBITUS exprime une quantité- la taille de l'espace utilisé - et REGISTRE une qualité : celle des régions occupées par les hauteurs constituantes de l'objet, à l'intérieur de cet espace déterminé, compte tenu des particularités acoustiques de l'instrument.

\subsubsection{AMBITUS}

Tout objet musical - horizontal ou vertical - occupe un espace borné à ses extrémités par sa hauteur la plus grave et sa hauteur la plus aiguë. Sa taille est donnée par la mesure de la distance séparant ces limites 18. Le résultat obtenu est une valeur absolue: un objet borné par Do3 et Do4 a un ambitus 12 (i.e. 12 demi-tons). L'ambitus relatif est la factorisation de ce résultat par rapport à l'espace total possible sur un piano conventionnel de 88 notes, c'està-dire 87. On obtient ainsi, non une valeur absolue, mais un tanx d'occupation de l'espace total disponible, ce qui permet une analyse comparée des différents objets en présence, dans cette dimension spécifique. Dans l'exemple précédent, l'objet d'ambitus 12 recevra une pondération de 0.1419 , ce qui représente un faible taux d'occupation de l'espace pianistique 20.

Mais AMBITUS ne discerne pas la ou les régions investies par l'objet à l'intérieur de cet espace déterminé. Or plusieurs invariants acoustiques, caractéristiques de l'instrument, tels que le matériau et le nombre de cordes, l'inharmonicité des graves ou encore la proportion de bruit dans l'extrême aigu, s'ajoutent aux variables contrôlées par la pensée à une utilisation sélective de cet 
espace -, pour en configurer une partition qualitative, de laquelle va dépendre significativement la qualité sonore finale de l'objet. C'est la raison pour laquelle une deuxième évaluation de l'écrit musical se fait nécessaire, cette fois sur la base de ce que nous avons appelé la registration.

\subsubsection{REGISTRE}

REGISTRE attribue un poids à chaque objet selon le nombre de régions de l'espace qu'il occupe, et selon la qualité sonore spécifique à ces régions. Pour ce faire, la tessiture du piano a été divisée en sept registres, comptés à partir d'un registre central de poids (0), délimité par les notes Fa3 et Fa5 21. Les registres audessous reçoivent la référence (-1) (Mi3-Sol\#2), (-2) (Sol2-Sil) et (-3) (La\#1-La0). Symétriquement, on a au-dessus de (0) les registres $(+1)$ (Fa\#5-Ré6), (+2) (Ré\#6-Do8) et (+3). (Ré\#-Do8). Ainsi, la totalité de la tessiture du piano est-elle décrite par l'ensemble contigus d'entiers $\{-3,+3\}$.

Après avoir évalué quels registres sont occupés, un poids leur est affecté, conformément à une loi de croissance de la valeur, proportionnelle à l'éloignement du registre central. En d'autres termes, plus l'objet occupe des registres éloignés du centre, plus sa valeur qualitative augmente, et réciproquement. Ces poids, invariables pour chaque registre, sont calculés de telle sorte qu'un objet occupant la iotalité de l'espace, c'est-à-dire les sept registres, va recevoir la valeur maximum (1). La coupure de l'échelle entre (0) et (1) est linéaire 22.

\subsubsection{ESPACE}

Le "composé" ESPACE 23 regroupe les informations fournies par AMBITUS et REGISTRE, par le simple calcul de la moyenne des deux pondérations reçues. Il s'agit d'une évaluation synthétique qui a l'avantage de prendre en compte simultanément l'aspect 
quantitatif de la configuration spatiale de chaque objet, et son aspect qualitatif, par une évaluation des modalités d'occupation de l'espace, évaluation pondéree en fonction $d$ un double critère de sonorité (invariables acoustiques) et de distribution (décisions compositionnelles locales).

\section{4. Évaluations}

Cfr. Fig. 2: Évaluations numériques de la séquence des taux d'occupation de l'espace (amb) et des régions sonores occupées localement (reg), dans La Cathédrale engloutie.

Commentaires:

$\mathrm{S}=$ sections macro-formelles ( $\mathrm{cf} f \mathrm{fi}$ )

$\mathrm{O}=$ étiquettes des objets discrets résultant de la méthode de segmentation. Les objets $\mathrm{O} 21$ et $\mathrm{O} 31$ ont été scindés en deux à cause de ruptures internes (cf. partition annexée); la scission améliore la fiabilité des évaluations. La section S0,5- ne contient qu'un objet unique, $\mathrm{O} 39$, en raison de l'extrême homogénéité sonore confrée par l'oscillation statique du dessin:flottant et sourd" das les graves. 24

mes: mesure dans laquelle débute l'objet Cette indication, figurant ici pour aider le lecteur au repérage sur la partition, est parfois très approximative, nombre d'objets ne débutant pas au début d'une mesure.

amb: liste des évaluations d'AMBITUS.

reg: liste des évaluations de REGISTRE.

esp: ESPACE (moyenne des deux colonnes précédentes).

\section{Analyse de l'ambitus relatif}

On a souvent observé que Debussy sollicitait fréquemment la quasi totalité de l'étendue du clavier. Les évaluations de AMBITUS corroborent cet aspect, pour ce prélude : l'ambitus relatif couvre une grande part de la tessiture 25. Mais plus intéressant est ce que nous montre le calcul de la corrélation entre les sections et la liste 


\begin{tabular}{|c|c|c|c|c|c|c|}
\hline $\mathrm{S}$ & 0 & mes & $\mathrm{amb}$ & reg & esp & commentaires \\
\hline 0 & 1 & 1 & 0.77 & 0.75 & 0.76 & $\mathrm{~S}=$ sections macro-formelles (cf fi.1) \\
\hline 0 & 2 & 3 & 0.79 & 0.75 & 0.77 & \\
\hline 0 & 3 & 5 & 0.8 & 0.75 & 0.77 & $\mathrm{O}=$ étiquettes des objets discrets résultant de la mé- \\
\hline 0 & 4 & 7 & 0.41 & 0.25 & 0.33 & thode de segmentation. Les objets $\mathrm{O} 21$ et $O 31$ ont étê \\
\hline 0 & 5 & 11 & 0.37 & 0.25 & 0.31 & scindés en deux à cause de ruptures intemes (cf \\
\hline 0 & 6 & 13 & 0.79 & 0.75 & 0.77 & partition annex $(e)$ ) la scission améliore la fiabilité \\
\hline 0 & 7 & 14 & 0.82 & 0.75 & 0.78 & des évaluations. La section S0,5-ne contient qu'un \\
\hline 0.25 & 8 & 16 & 0.6 & 0.5 & 0.55 & objet unique. O39, en raison de l'extrême homo- \\
\hline 0.25 & 9 & 17 & 0.79 & 0.75 & 0.77 & généité sonore conféré par l'oscillation statique \\
\hline 0.25 & 10 & 18 & 0.79 & 0.75 & 0.77 & du dessin "flottant et sourd" dans les graves 24 \\
\hline 0.25 & 11 & 19 & 0.69 & 0.75 & 0.72 & \\
\hline 0.25 & 12 & 20 & 0.6 & 0.58 & 0.59 & \\
\hline 0.25 & 13 & $21 \mathrm{a}$ & 0.74 & 0.58 & 0.66 & mes : mesure dans laquelle débute l'objet. Cette in- \\
\hline 0.25 & 14 & $21 \mathrm{~b}$ & 0.71 & 0.5 & 0.61 & dication, figurant ici pour aider le lecteur au repe- \\
\hline 0.25 & 15 & 22 & 0.69 & 0.67 & 0.68 & rage sur la partition, est parfois très approximati- \\
\hline 0.25 & 16 & 23 & 0.49 & 0.25 & 0.37 & ve, nombre d'objets ne débutant pas au début d'une mesure. \\
\hline 0.25 & 17 & 25 & 0.55 & 0.5 & 0.52 & \\
\hline 0.5 & 18 & 28 & 0.63 & 0.58 & 0.61 & amb : liste des evvaluations d' AMBIT US. \\
\hline 0.5 & 19 & 32 & 0.61 & 0.5 & 0.56 & \\
\hline 0.5 & 20 & 34 & 0.67 & 0.58 & 0.62 & reg : liste des évaluations de REGISTRE \\
\hline 0.5 & 211 & 38 & 0.61 & 0.5 & 0.56 & \\
\hline 0.5 & 212 & 40 & 0.57 & 0.5 & 0.53 & esp : ESPACE (moyenne des deux colonnes précé- \\
\hline 0.5 & 22 & 42 & 0.74 & 0.5 & 0.62 & dentes). \\
\hline 0.5 & 23 & 43 & 0.74 & 0.5 & 0.62 & \\
\hline 0.5 & 24 & 44 & 0.76 & 0.5 & 0.63 & \\
\hline 0.5 & 25 & 45 & 0.76 & 0.5 & 0.63 & \\
\hline 1 & 26 & 46 & 028 & 0.25 & 0.26 & \\
\hline 1 & 27 & 51 & 0.36 & 0.25 & 0.31 & \\
\hline 1 & 28 & 53 & 0.63 & 0.42 & 0.52 & \\
\hline 1 & 29 & 55 & 0.67 & 0.5 & 0,59 & \\
\hline 1 & 30 & 57 & 0.69 & 0.5 & 0,6 & \\
\hline 1 & 311 & 59 & 0.75 & 0.42 & 0,58 & \\
\hline 1 & 312 & 60 & 0.72 & 0.5 & 0,61 & \\
\hline 1 & 32 & 61 & 0.75 & 0.42 & 0,58 & \\
\hline 1 & 33 & 63 & 0.36 & 0.08 & 0.22 & \\
\hline 1 & 34 & 64 & 0.33 & 0.25 & 0,29 & \\
\hline 1 & 35 & 65 & 0.47 & 0.25 & 0,36 & \\
\hline 1 & 36 & 68 & 0.07 & 0.42 & 0,24 & \\
\hline 1 & 37 & 69 & 0.07 & 0.42 & 0,24 & \\
\hline 1 & 38 & 70 & 0.09 & 0.42 & 0.25 & \\
\hline 0.5 & 39 & 72 & 0.53 & 0.5 & 0,51 & \\
\hline 0 & 40 & 84 & 0.74 & 0.58 & 0.66 & 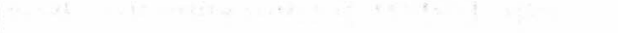 \\
\hline 0 & 41 & 85 & 0.91 & 1 & 0,96 & \\
\hline 0 & 42 & 86 & 0.91 & 1 & 0,96 & \\
\hline 0 & 43 & 87 & 0.91 & 1 & 0,96 & \\
\hline
\end{tabular}

Fig. 2: Évaluations numériques de la séquence des taux d'occupation de l'espace $(\mathrm{amb})$ et des régions sonores occupées localement (reg), dans La Cathédrale engloutie. 
des évaluations de AMBITUS (fig. 3) : c'est que ce vaste espace est exploité de manière systématique lors des sections liminaires (axes verticaux S0+ et S0- dans la figure) 26, qu'il se concentre au niveau des sections médianes (soit les deux S0.5), pour connaitre une relative réduction dans la section centrale (axe vertical S1) 27.

On peut, à partir de ce comportement, fonder l'hypothèse d'une articulation des ambitus en rapport dialectique avec l'archétype formel, en vertu de laquelle le mouvement dynamique ascendant qui aboutit à la section centrale, c'est-à-dire au climax, se caractérise par une compression de l'espace utilisé.

Puis, à mesure que la dynamique de l'oeuvre redescend vers la section terminale, l'espace s'élargit jusqu'à son maximum. Autrement dit, le profil de l'occupation spatiale adopterait une forme en opposition symétrique à la forme globale, comme le montre la fig. 4 .

On voit que la courbe d'évolution des taux d'occupation de l'espace pianistique simule bien une forme concave qui interagit dialectiquement sur la convexité macro-formelle.

L'observation des modalités d'utilisation des registres va compléter ces premières données.

\section{Analyse de la distribution des sons à l'intérieur des ambitus observés}

Dans le graphique ci-dessous (fig. 5), les listes des évaluations effectuées par AMBITUS et REGISTRE ont été représentées sous forme de courbes montrant la variation des valeurs respectives. Bien que globalement parallèles, on remarque qu'elles divergent en plusieurs points :

Il est difficile de ne pas constater que les divergences les plus remarquables et les plus durables se concentrent dans les deux sections "thématiques" ascendantes S0.5+ et S1. Cette caractéristique suggère qu'un des attributs de la sonorité comme élément de signature thématique pourrait résider dans les relations entre l'espace total utilisé et la distribution des sons à l'intérieur de 


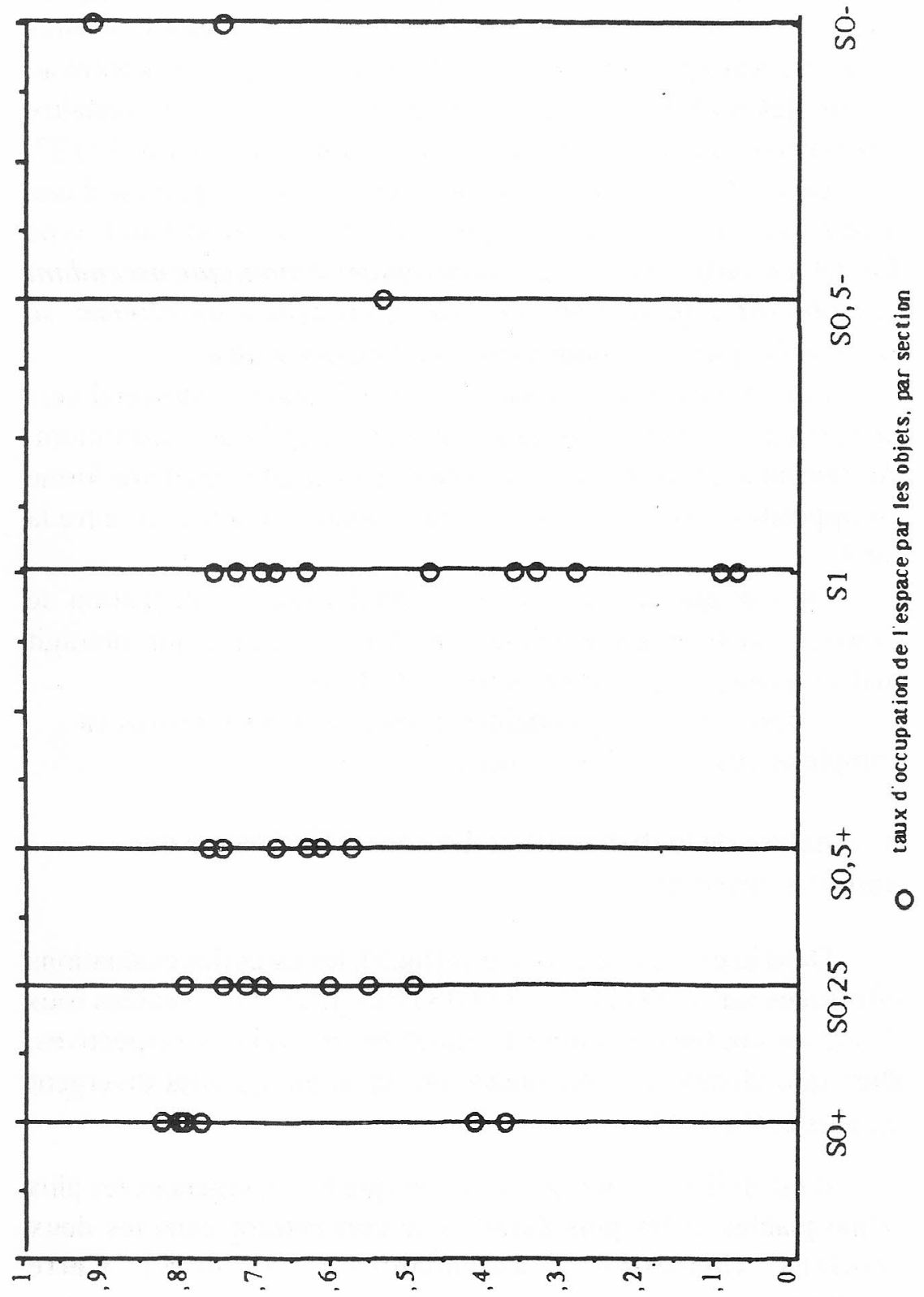

Fig 3: Corrélations - Sections macro-formelles/Ambitus relatifs 


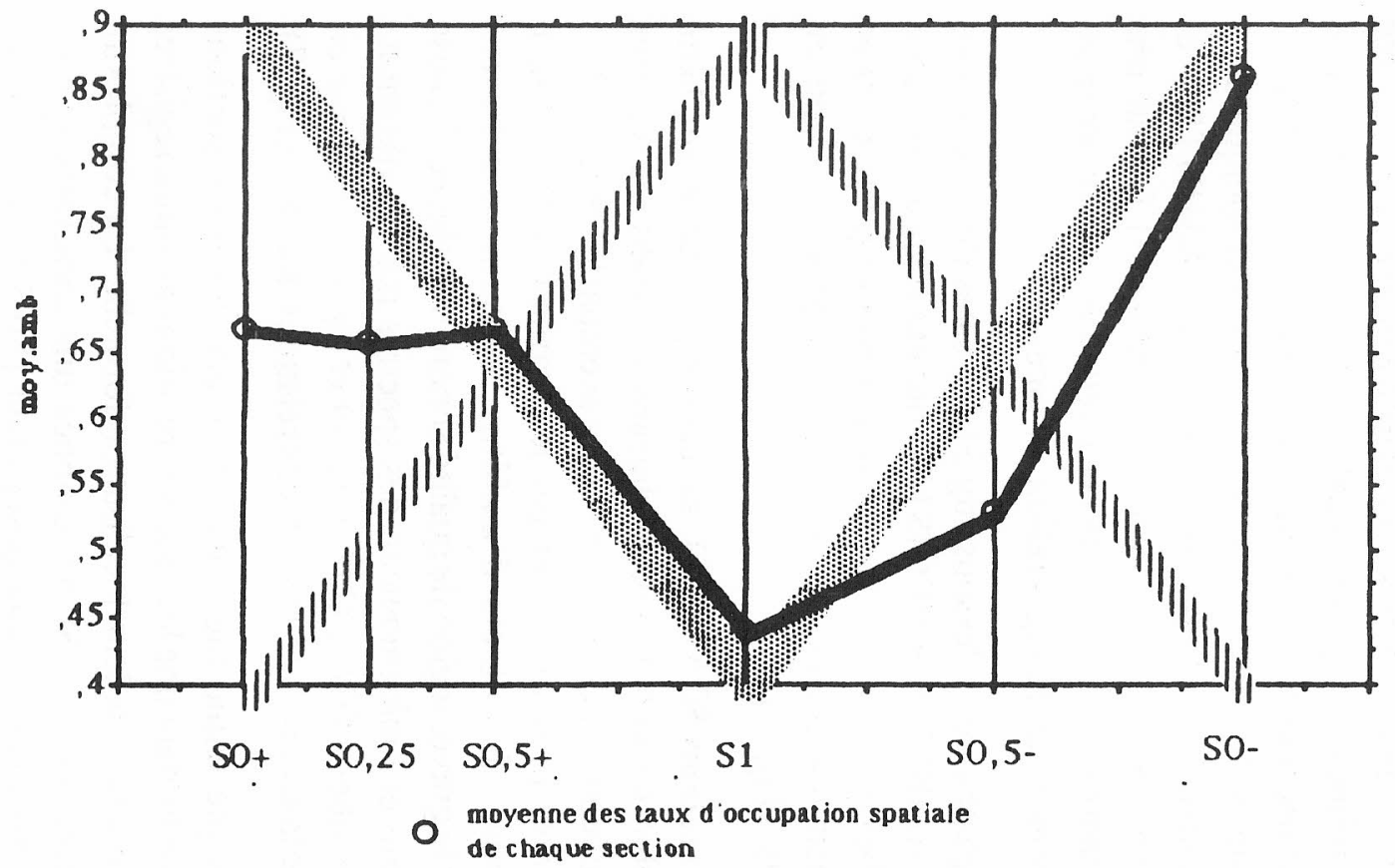

schématisation de la courbe de l'evolution des taux

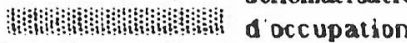

|IIIIIIIIIIIIIIIII schemat isation de la courbe macro-formelle 
cet espace. Dans les sections liminaires, la distribution des sons apparaît comme étant directement proportionnelle à l'espace total occupé par l'objet : plus l'ambitus de l'objet est grand, plus il occupe les registres extrêmes - de grande "qualité sonore" - en remplissant simultanément les registres intermédiaires : voir par exemple les premières mesures de l'oeuvre. La représentation graphique de la corrélation entre les valeurs retournées par AMBITUS et REGISTRE pour S0 (les deux versants confondus) fournit une image très parlante de la linéarité de cette relation : à petit ambitus, faible qualité sonore de la registration, et vice-versa.:

Dans le graphique ci-dessous (fig.5), les listes des évaluations effectuées par AMBITUS et REGISTRE ont été représentées sous forme de courbes montrant la variation des valeurs respectives. Bien que globalement parallèles, on remarque qu'elles divergent en plusieurs points: (Fig. 5).

Dans les sections $\mathrm{S} 0.5+$ et $\mathrm{S} 1$, la relation perd cette simplicité linéaire : des objets à ambitus non négligeable occupent les registres de basse ou moyenne "qualité sonore" (par exemple les objets O32 à 035, m. 61 et sq.), excluant souvent les extrêmes (O22 et sq.). Réciproquement, des objets d'ambitus restreint investissent exclusivement le grave, région de qualité sonore très élevée, à cause de la complexité et l'inharmonicité des spectres mis en vibration; c'est le cas des objets $\mathrm{O} 36$ à $038, \mathrm{~mm} .69$ et sq. Le graphique cidessous illustre la complexité relative des relations dans S1: (Fig. 7).

On remarque enfin que sous cet aspect, les deux sections intermédiaires adoptent une logique des relations ambitus/registres différente en fonction de leur localisation formelle. La complexité des rapports trouvés dans S0.5+ annonce une caractéristique de $\mathrm{S} 1$, alors que dans son versant descendant (S0.5-), ces relations calquent la linéarité propre aux sections liminaires (S0). Sur ce plan, S0.5-est bien un miroir de S0.5+. Nous pouvons donc dire que les modes de distribution spatiale sont un des éléments de l'écriture contribuant à caractériser la qualité sonore de ces deux groupes de sections. 


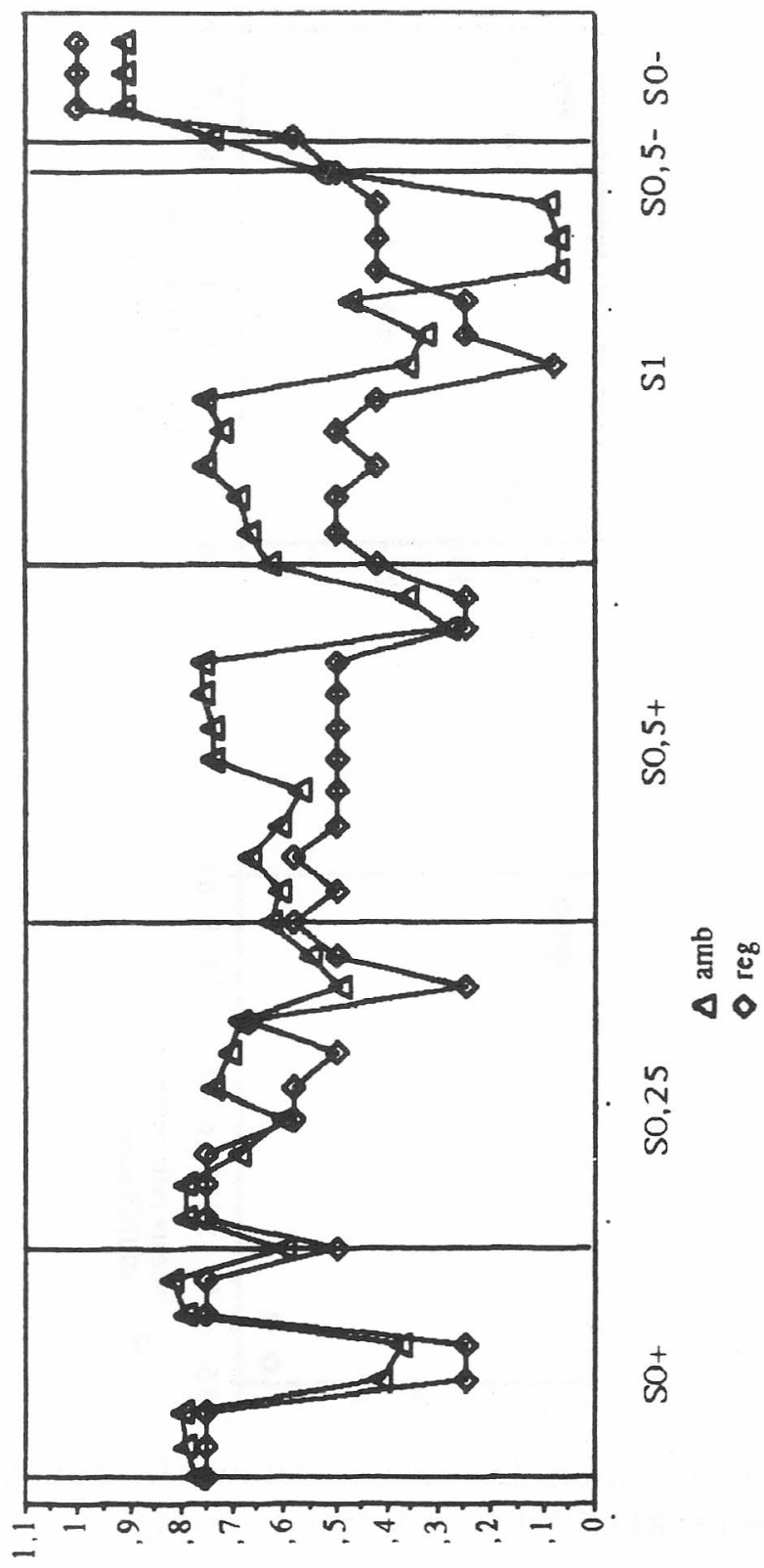

Fig. 5: Courbes représentant la séquence des taux d'occupation de l'espace et les registres investis. 

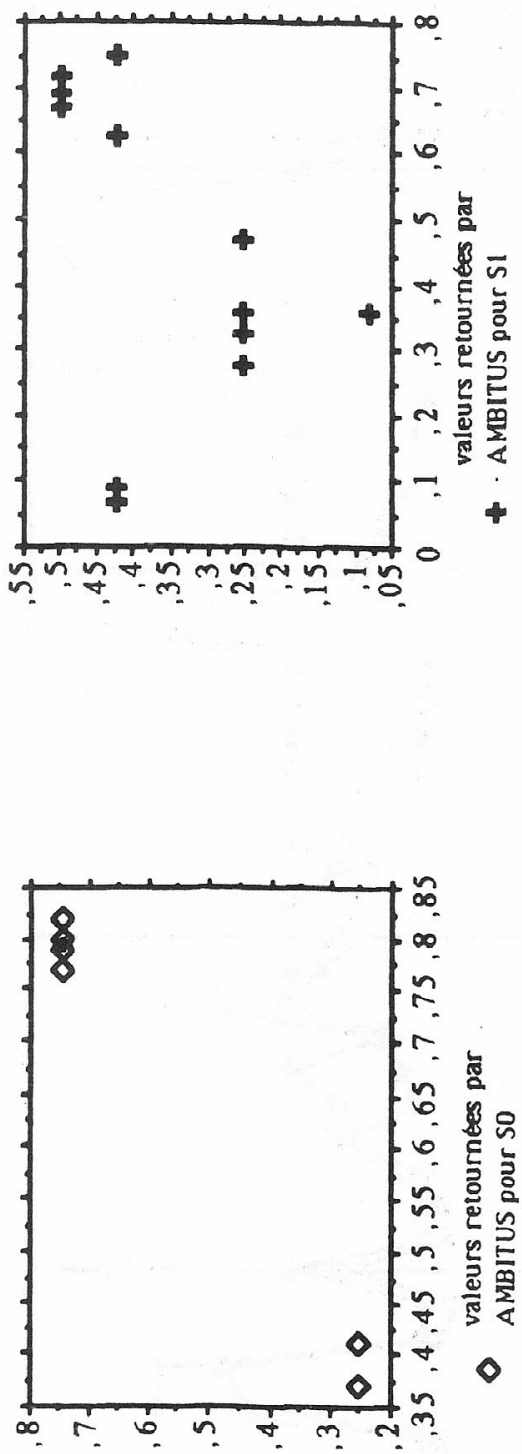

Fig. 6: Corrélation des valeurs retournées par AMBITUS (abscisses) et REGISTRE (ordonnées), pour S0.

Fig. 7: Corrélation des valeurs retournées par AMBITUS (abscisses) et REGISTRE (ordonnées), pour SI (comparer. avec la fig. 6). 


\section{Espace et structure thématique}

La contribution du composant REGISTRE à l'analyse formelle est également très significative si l'on utilise les informations complémentaires qu'il est en mesure de nous fournir, dont voici dans le tableau ci-dessous la liste: (Fig. 8).

Col. 1: numéro d'ordre des objets, suivi, pour chaque objet, du taux d'occupation de chacun des 7 registres (en nombre de notes présentes dans chacun d'entre eux). Les registres sont rangés du plus grave (-3) à gauche au plus aigu $(+3)$ à droite.

Col. 2: taux d'occupation de chacun des 7 registres, en réponses "oui" (1) ou "non" (0). Même rangement des registres que pour la $\mathrm{col} .2$, le premier bit à gauche informant le registre -3 , etc

Col. 3: nombre de registres occupés par l'objet $(\{1,7\})$.

Col. 4: Registre contenant le plus grand nombre de notes pour chaque objet. Le premier chiffre indique ce registre $(\{-3,3\})$ et le deuxième le nombre de notes qu'il contient

Col. 5: Rappel du contenu motivico-thématique : M, motif de base, TS, "thème-sujet en Do\#", TO, "thème-objet en Do".

Les cadres délimitent les six sections formelles.

Concentrons-nous sur la col 2. En la parcourant visuellement de haut en bas, défile la séquence des distributions spatiales du Prélude. Observons les différences de distribution entre TS. TO et M.

\subsection{Le thème-objet, stasis spatiale dans le grave}

TO, lors de ses deux apparitions (ci-dessus, O 18 à 21 , puis O39), revendique une indéniable identité sonore en occupant immuablement et pleinement la même étendue située dans la partie grave de l'instrument $(\{-3,0\})$. Il est intéressant de remarquer que la seule excursion vers le registre $+1(\mathrm{O} 20)$ est un des éléments d'écriture qui configure le climax propre à l'évolution de TO dans 


\begin{tabular}{|c|c|c|c|c|}
\hline 1 & 2 & 3 & 4 & 5 \\
\hline 112210430 & 1111110 & 6 & 010 & $\overline{\mathrm{M}}$ \\
\hline 212210430 & 1111110 & 6 & 010 & $M$ \\
\hline 312210330 & 1111110 & 6 & 010 & $\mathrm{M}$ \\
\hline 400010330 & 0001110 & 3 & 010 & TS \\
\hline 500012420 & 0001110 & 3 & 012 & TS \\
\hline 62213120 & 1111110 & 6 & 03 & $\mathrm{M}$ \\
\hline 72239420 & 1111110 & 6 & 09 & $\mathrm{M}$ \\
\hline 81229000 & 1111000 & 4 & 09 & $\bar{M}$ \\
\hline 91229220 & 1111110 & 6 & 09 & $\mathrm{M}$ \\
\hline 101229220 & 1111110 & 6 & 09 & $\mathrm{M}$ \\
\hline 1121211110 & 1111110 & 6 & 011 & M \\
\hline 1221211100 & 1111100 & 5 & 011 & M \\
\hline 1331410100 & 1111100 & 5 & 010 & M \\
\hline 1431411000 & 1111000 & 4 & 011 & M \\
\hline 151209410 & 1101110 & 5 & 09 & M \\
\hline 1600011570 & 0001110 & 3 & 011 & M \\
\hline 175648000 & 1111000 & 4 & 08 & $\mathrm{M}$ \\
\hline 185648000 & 1111000 & 4 & 08 & TO \\
\hline 1913514000 & 1111000 & 4 & 014 & TO \\
\hline 2012515300 & 1111100 & 5 & 015 & TO \\
\hline 21111515000 & 1111000 & 4 & 015 & TO \\
\hline 2121127000 & 1111000 & 4 & 07 & $\mathrm{M}$ \\
\hline 221016320 & 0111110 & 5 & 06 & M \\
\hline 231016320 & 0111110 & 5 & 06 & M \\
\hline 241016320 & 0111110 & 5 & 06 & M \\
\hline 251016320 & 0111110 & 5 & 06 & $\mathrm{M}$ \\
\hline 261061000 & 0111000 & $\overline{3}$ & -16 & $\overline{T S}$ \\
\hline 271065000 & 0111000 & 3 & -16 & TS \\
\hline 28100010310 & 0101110 & 4 & 010 & TS \\
\hline 291037320 & 0111110 & 5 & 07 & TS \\
\hline 301038430 & 0111110 & 5 & 08 & TS \\
\hline 3111009340 & 0101110 & 4 & 09 & TS \\
\hline 3121028440 & 01111110 & 5 & 08 & TS \\
\hline 3210011450 & 0101110 & 4 & 011 & TS \\
\hline 3300619000 & 0011000 & 2 & 019 & TS \\
\hline 340149000 & 0111000 & 3 & 09 & TS \\
\hline 3510311000 & 0111000 & 3 & 011 & TS \\
\hline 362100000 & 1100000 & 2 & -32 & \\
\hline 372100000 & 1100000 & 2 & -32 & \\
\hline 382200000 & 1100000 & 2 & -32 & \\
\hline 3922512000 & 1111000 & $\overline{4}$ & 012 & TO \\
\hline 4010212510 & 1011110 & 5 & 012 & $\bar{M}$ \\
\hline 4121212541 & 1111111 & 7 & 012 & $\mathrm{M}$ \\
\hline 422117131 & 1111111 & 7 & 07 & $M$ \\
\hline 432117131 & 1111111 & 7 & 07 & M \\
\hline
\end{tabular}

Fig. 8. Liste complémentaire d'informations retournees par REGISTRE: 
S0.5+, mm.36-37 (notes Sol5-La5-Sib5 28). Cette unique expansion de l'espace - associée à un crescendo dont on doit souligner qu'il amène au niveau d'amplitude le plus élevé de toute l'oeuvre - nous semble sans aucun doute l'agent essentiel de l'expression de ce climax. En effet, on ne peut dire que la structure de la mélodie per $s e$, et moins encore celle de l'harmonie, favorisent un mouvement vers la tension. Serge Gut 29 a non seulement clairement démontré les raisons de l'extrême statisme de l'harmonie à cet endroit-là. comme il a attire l'attention sur le fait que le compositeur évite soigneusement l'apparition du triton mélodique -- élément essentiel de tension - en substituant chaque fois que c'est nécessaire un Sib à la sensible Si bécarre.

Quant aux configurations rythmiques, elles n'evoquent ni ne suscitent aucune accélération dynamique, mais plutôt le contraire : il suffit d'observer que la plus grande variété rythmique se trouve concentrée dans le segment d'ouverture (O18), et qu'elle tend par la suite à s'immobiliser dans la régularité de la pulsation 30 . Il semble donc que le compositeur ait exclusivement réservé l'écriture de ce mouvement de tension aux dimensions espace et amplitude. c'està-dire à des dimensions propres au timbre.

\subsection{Le thème-sujet, dynamique vers l'aigu}

TS, pour sa part, se différencie nettement de TO dans sa mouvementation spatiale: n'utilisant jamais le registre -3 , il se déploie plus facilement vers +1 et même +2 à un "thème-objet" centré sur le grave de l'instrument, semble répondre un "thèmesujet" exploitant les autres régions de l'espace. Mais l'articulation de cet espace est suffisamment subtile pour qu'on ne se contente de ces constatations générales, d'ailleurs fréquemment relevées dans la littérature.

Le premier énoncé de TS, dans $\mathrm{S} 0+(\mathrm{O} 4-5)$ est exclusivement concentré dans le médium-aigu $(\{0,+2\})$. Dans le contexte local, ceci représente un soudain et très sensible amenuisement de l'espace par rapport aux événements qui l'entourent, lesquels évoluent dans 
la tessiture $\{-3,+2\}$. En outre, la suspension des vibrations profondes des graves, qui se concrétise aux cours des mm.5-6 quand les résonances de la partie "main gauche" de l'accord s'estompent, au profit des sonorités en octaves dans le médium-aigu des mm.67, constitue une transition timbrale fondamentale pour l'articulation des plans sonores et pour l'instauration progressive de la sonorité "douce et fluide" du thème-sujet qui émerge alors.

Quand TS est réinstauré, m.47(O26), il l'est exactement dans une région de registres symétriquement opposée à celle du premier énoncé : à savoir $\{-2,0\}$, au lieu de $\{0,+2\}$ la première fois. Il en résulte une sonorité totalement nouvelle : indépendamment de la hauteur, le timbre du registre médium-grave possède des caractéristiques notablement différentes de celui du médium-aigu 31. En mettant en relation deux registres de'l'instrument bien identifiables, et en articulant cette relation dialectiquement à l'organisation thématique, qui reste pour l'essentiel invariable 32. le compositeur confère à cette dimension un statut d'élément souverain de l'expression de la forme.

Cette deuxième occurrence de TS connait ensuite une très importante expansion jusqu'au registre $+2(\mathrm{O} 28-32)$. Nous sommes ici au climax formel de l'oeuvre, et TS est proprement développéau sens spatial du terme, qui n'a jamais éte si judicieux - de manière à couvrir tout l'espace $\{-2,+2\}$, qu'il n'avait jusqu'alors occupé qu'en alternance. Puis, quand intervient ce que Schoenberg appellerait la phase de «liquidation » 33 (mm.62 et sq., O33-35), l'espace se replie pour recréer exactement, mm.64 à 67, la région que nous avions au départ : $\{-2,0\}$. TS est ici terminé, mais la phase de repli vers le grave se poursuit encore dans les mesures de transition annonçant la reprise de TO : les mm.68-71 (O36-38) se caractérisent par une utilisation exclusive de $\{-3,-2\} 34$. La fig. 9 reproduit les courbes de l'évolution de la registration et des amplitudes dans S1 35:

La curvilinéarité les deux dimensions est pratiquement parallele. Dans la phase ascendante de la courbe. l'expansion de l'espace, à partir de la m.53, prépare l'augmentation de l'amplitude, 


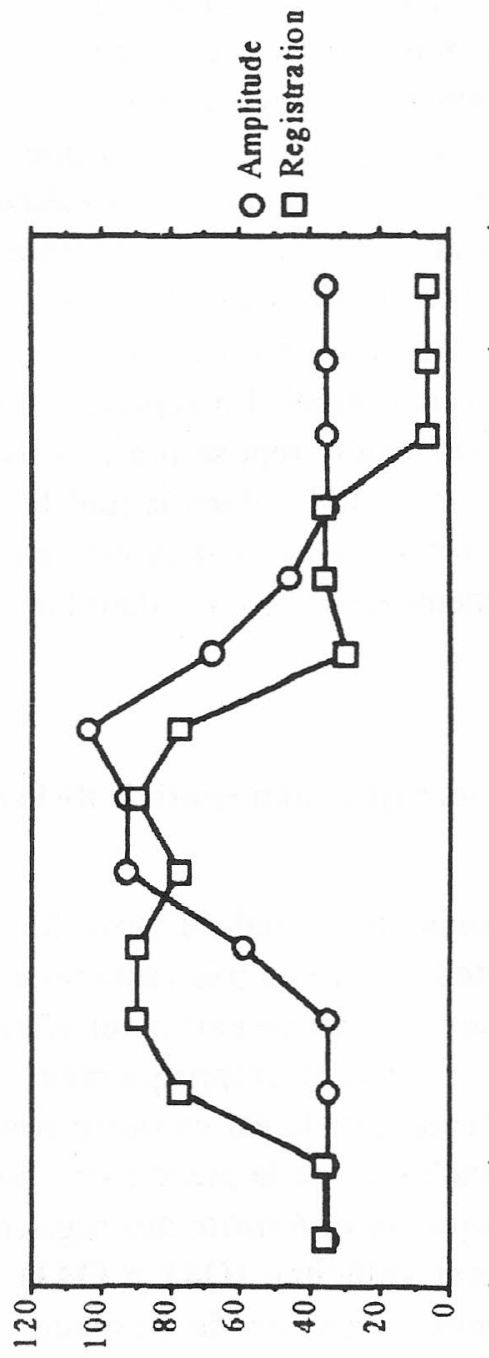

Fig. 9: Courbes d'évolution des amplitudes et registrations dans S 1. 
qui débute trois mesures plus tard. Les deux dimensions se conjuguent alors pour créer le climax central de l'oeuvre ( $\mathrm{mm}$.5962). Puis, dans la phase de liquidation, la restriction assez abrupte de l'espace utilisé - correspondant à la fameuse séquence de septièmes parallèles, mm.62-64 - accompagne en l'emphatisant le retour aux faibles amplitudes originales. Ce comportement intercohérent permet de soutenir l'existence d'une conjonction des dimensions "amplitude" el "espace" dans l'écriture de la forme de S1. Cette conjonction devient un facteur primordial de configuration formelle, sur laquelle s'appuie le développement du thème-sujet. Cette configuration n'est autre que la reproduction locale du modèle macro-formel. En reproduisant l'archétype de simétrie dynamique sur lequel repose toute la pièce, S1 offre un espace unique dans le prélude, dans lequel le compositeur va pouvoir, fait assez rare pour avoir déjà été relevé, instaurer un authentique développement thématique, dans l'acception classique de ce concept.

\subsection{Le motif de base, expression spatiale de la monumentalité}

Quant au déploiement spatial du motif $M$, qui dessine dès la première mesure de l'oeuvre un très vaste territoire $(\{-3,+2\})$, il contribue pour une très grande part à cet effet de profondeur et de mommentalité souvent empiriquement évoqué par les musicologues 36 . Remarquable est en outre son retour, lors de la courte section conclusive de la pièce ( $\mathrm{SO}-, \mathrm{mm} .84$ et sq.) : il configure le lieu unique où la totalité des registres du piano ( $\{-$ $3,+3\})$ est finalement sollicitée (041 à O43). S0- clôt cette oeuvre par une ultime extension de l'espace, qui atteint aux limites de l'instrument (Dol-Sol7 37). Si l'on a pu voir dans $L a$ Mer l'essence d'une forme ouverte 38, l'écriture de l'espace dans La Cathédrale engloutie exemplifie une forme qui, au moment où elle se replie sur elle-même, ouvre l'espace sonore sur un quasi-infini. 


\section{Espace versus structure formelle}

Les observations qui précédent dirigent notre analyse vers des conclusions mettant en évidence une relation coordonnée entre l'utilisation de l'espace pianistique et les différentes entités thématiques de l'oeuvre. Mieux, la dialectique abstraite que nous avons suggérée implicitement, à la suite d'autres auteurs, entre, d'une part, un thème "objet", statique, et un thème "sujet", mouvant, et d'autre part, des sections "thématiques" centrales, et des sections "motiviques" liminaires, se trouve vérifiée dans cette dimension. Il nous semble d'ailleurs tout à fait justifié d'inverser la formulation et de suggérer que ce sont les unités motivico-thématiques qui s'insèrent a posteriori dans une articulation formelle structurée $a$ priori par l'écriture de l'espace, et non le contraire.

Les termes principaux de cette inter-relation sont que $\mathrm{TO}$ et TS se définissent par une stratégie opposée d'occupation de la tessiture de l'instrument, et que sections liminaires et centrales se distinguent par une relation spécifique à l'espace total utilisé et à la distribution des sons à l'intérieur de cet espace.

En synthèse, il existe une forte corrélation négative entre le découpage formel de l'oeuvre tel que l'a suggéré Debussy par ses indications paratextuelles, formalisées ensuite par nous-même à partir de la proposition de Roy Howat, et l'écriture de l'espace, interprétée comme un des éléments de l'écriture des sonorités : le très ample champ spatial et sonore qui ouvre la pièce se comprime à mesure que la forme avance vers son point culminant, tandis que la dynamique dépressive qui caractérise la partie finale de l'oeuvre est accompagnée d'un mouvement contradictoire d'expansion maximum de ce champ.

Ce n'est pas tout : les sections médianes - étiquetées S0.5-, se caractérisent par des valeurs spatiales également médianes, avec très peu de dispersion autour de ces moyennes. Comparée à $\mathrm{S} 0$ et $\mathrm{S} I$, la section S0.5 - lieu où s'énonce le "thème-objet" - forme un contraste structurel très net, sous cet aspect aussi. On en conclura que l'écriture des constituants de base du discours musical - 
hauteurs, durées - est indissociablement liée à une définition précise de l'espace dans lequel ils vont évoluer, et qu'une fois déterminés, les attributs qualitatifs de cet espace deviennent des invariants de l'écriture.

\section{Conclusion}

On peut donc retenir de cette série d'évaluations statistiques que l'étude objective des modalités d'utilisation de l'espace dans cette oeuvre nous fournit des informations non dénuées d'intérêt quant à l'appréhension des stratégies debussyennes concernant l'expression de la forme au moyen de dimensions relevant de l'écriture de la sonorité. Quand l'on sait qu'il fut le premier à les exploiter systématiquement, en tant que composants autonomes de la pensée musicale, qui ne soient pas assujettis aux lois d'organisation des cellules élémentaires que sont les notes individuelles 39 , on ne doutera pas qu'une investigation approfondie de son oeuvre, à l'aide d'outils analytiques spécifiques, puisse apporter des éléments permettant enfin une analyse non métaphorique de la technique de composition des sonorités propre à Debussy, et sur les moyens par lesquels s'exprime son immense originalité.

Paris, Nov. 1993/ João Pessoa, Août 1994. 


\section{NOTES}

1. Les citations sont respectivement de Jankélévitclr 1968. Charru 1988. Schmitz 1966 |ce dernier dans notre traductionl.

2. JAROCINSKY, S.. 1970 : Debussy, impressionisme et symbolisme. Scuil. Paris.

3. Voir par excmple: LOCKSPEISER. E. 1980: Claude Dehussy, Fayard. Paris: GUT. S., 1986: "La Cathédrale engloutic. prélude de Claude Debussy : interférences entre le matériau. la structure et la forme", Analyse Musicale $1^{\circ}+$, pp. 78-81: CHARRU. P. 1988 : "Les 24 Préludes pour Piano de Debussy : Ic mouvement musical au rythme de la forme". Analywe Musicale $\mathrm{n}^{\circ} 12$, pp. 63-88; $\mathrm{el}$ al..

4. PASCOAL. M.L. 1989 : Prelúdios de Debussy : reflexo e projeção, Thèse de Doctorat, Universidade Estadual de Campinas. São Paulo: JAROCINSKY op.cit., et al.. Ces relatives faiblesses dans ce domaine particulier n'invalident en rien la qualité de ecs travaux quant à la connaissance de l'oeuvre de Debussy d'une manière générale.

5. André Souris a pu ćcrire que «l'analyse traditionnclle, consistant à valoriser de soi-disant "themes" ou de soi-disant schémas tonaux, n'aboutit ici qu'à de médiocres résultats " [SOURIS, A., 1965 : "Poćtique musicale de Debussy", in WEBER. É. (éd.) : Dehussve l'évolution de la musique au .LTè siècle. C.N.R.S.. Paris. p.137!

6. HOWAT, R. 1983 : Debussy in proportion, a musical analysis. Cambridge U.P. Cambridge. Les pages 159 à 162 sont consacrécs à La Cathédrale engloutie.

7. Voir en particulier lc Chapitre II de cet ouvrage. Jarocinsky op.cit laisse penser que. si Debussy a pu éprouver de la "curiosité " pour les phénomènes relevant de l'étrange ou de l'occulte. il n'a cu que de « très brefs » contacts avec ce milicu.

8. Ce motif est constitué de la cellule de trois sons formée des intervalles de 2d majeure +5 tc juste lcf RETI. R.. 1978 : The Thematic Process in Music. Greenwood Press. Westport [réimpression de 1951. MacMillan|. pp. 1942061

9. Le "miroir" concerne non le matériau élémentaire de l'écriture (hauteurs et durées), qui est identique dans les deux présentations. mais celui qui configure le timbre (intensités. densités).

10. Elle est dominante non seulement dans le sens où elle constitue une métaphore du sommet de la voûte, mais aussi d'unc certaine manière dans le sens où la région dominante se définit, plus que par une tonalité donnéc. par sa capacité à générer une tension par rapport à la région tonique [cf. GUT op.cit.]. 
11. Lui-même également issu du motif de base initial.

12. "S" pour "section"

13. BARRAQUE, J. 1965: "Debussy ou l'approche d'unc organisation autog_ne de la composition", in Weber. E. (ćd.), 1965: Debussy et l'évolution de la musique au .lYè siécle. C.N.R.S. Paris. pp. 83-96.

14. Le pôle harmonique sur lequel il ćvolue est lui aussi oscillatoire, entre le Mi et le Sol\# (cr Gut op.cit. :80).

15. Serge Gut op.cit. adopte implicitement cet angle d'analyse : il appelle l'un - Ic TO - "thème principal" et l'autre "thème secondaire".

16. C'est pourtant la méthode de saisie adoptée par Holden dans son travail sur les tcxtures. comme si la mesure était pour Debussy un marqueur significatif dans cette dimension (HOLDEN, C.E., 1973 : The onganisation of texture in selected piano compositions of Claude Dehussw. Ph.D.. University Microfilms International. Ann Arbor. Michigan!.

17. GUIGUE, D.. 1995 : Thèse de Doctorat en Musicologic du XXème sic̀cle, en préparation. Elle se base en partic sur Ies connaissances que la recherche psychoacoustique a apportée sur la cognition auditive humaine Icf. par cxcmple McADAMS, S.. \& BIGAND. E.. 1994: Penser les sons: Psychologie cognitive de l'audition. P.U.F., Paris|

18. Pour calculcr cette distance, on aura préalablement cncodé numériquement Ics notes. Nous avons adopté une numération conforme à la norme Midi. où 21 correspond au La grave (La 0) du piano, 60) au Do central (Dot) ct 108 au dernicr Do (Do8). Les registres sont numérotés selon le système américain. ou Lat est le $\mathrm{La}(4+0 \mathrm{~Hz})$ du diapason.

19. c'est-à-dirc 12/87.

20. le taux maximum d'occupation étant 1 .

21. L'espace nous manque dans cet exposé pour justifier ces choix (ils le sont in GUIGUE 1995). Ils se fondent sur les informations rapportécs dans nombre d'articles ou ouvrages relatifs à l'acoustique de l'instrument, en particulier en ce qui concerne la facture el le nombre des cordes. la présence ou non d'étouffoirs. et le phénomène de stretching. Un article de SUZUKI. H. \& NAKAMURA, I., (1990) : "Acoustics of Piano". Applied Acoustic:s $\left.n^{\circ} 30\right]$ fait un point objectif sur la question.

22. L'implémentation informatique permet ćgalement de recevoir d'autres informations relatives à la registrtion : Ic taux d'occupation de chaque registre, le registre le plus occupé ctc...

23. Le terme de "composé", que nous devons à Philippe Manoury a été choisi à cause du lien sémantique qui le relic à l'essence de l'acte de "composer" manipuler, articuler des objets et des dimensions complexes. [MANOURY. P. 1991 :"Les limites de la notion de 'timbre' ". in BARRIERE. J.B. (éd), 1991: Le timbre, métaphore pour la composition, Bourgois. Paris|. 
24. Jankélévitch op.cit. a associć ce type d'écriture à une obsession de la stagnation de l'eau chez. Debussy. Cette immuabilité. ce moment de suspension du mouvement, est créc par la vibration sonore permanente entretenue par la basse. alliće au statisme du thème-objet en écho. Ces caractéristiques plaident pour l'unification de toute la section en une seule entité. à l'inverse de son premier énoncé sur le versant ascendant (SO).5+). dans lequel les accents de la basse déterminent de nettes ruptures de continuité.

25. Moyenne des ambitus relatifs (i.c. moyenne de la liste "amb" de la fig.2) : 0.61. En d'autres termes, cette valeur signifie que $61 \%$ de la tessiture du piano est occupćc. Plus forte taxe d'occupation trouvéc pour un objet isolé : 0.91 : plus petite taxe 0.07 .

26. Moyenne des valcurs de $A M B$ pour $S 0+e t S O$ - confondues : 0.75 ; valeur minimum observéc : 0.37 . Notons que les deux valeurs les plus basses observées dans $\mathrm{S}(0+$ les deux points les plus bas de l'axe vertical de la figure) se référent à la présence du "thème-sujet" mes.7 ct sq., thème qui n'est pas repris dans $\mathrm{S}($ )-

27. Moyenne des valeurs de AMB pour S1 : 0.44 : valeur minimum : 0.07 . On observera en outre qu'en contrepartic. cette section centrale connait la plus grande dynamique spatiale : Ies taux d'occupation sont plus variés que dans les autres sections.

28. Le Sol5 apparaît déjà unc unique fois dans O18 Cette exception statistique l'a éliminé de l'évaluation informatique. Dans O20. Ic Sol5, répété 3 fois, constitue par contre une des notes-pivot de la mélodic à la voix supéricure.

29. GUT, S., 1981 : "Les techniques d'harmonic impressionnistc chez Debussy", Revue Internationale de .Musique Française n ${ }^{\circ} 5$, pp. 31-42.

30. La densité verticale. autre composant essentiel pour l'écriture de la sonorité, est également significativement invariante dans TO.

31. Ces différences de timbres sont provoquées en grande partic par des caractéristiques de facture instrumentale : I ou 2 cordes filetées dans le grave, 3 cordes non filetées dans l'aigu. Une autre particularité est que le taux de désaccord (detuning), élément important de la sonorité du piano. est naturellement plus important entre 3 cordes qu'entre 2 cordes, et que ceci entraîne des différences dans le comportemeṇt acoustique du son. notamment le laux ct les modalités de son extinction.

32. On aura bien sûr remarqué le changement de la note-pédale (Sol\# grave au lieu de Mi aigu dans le premier énoncé). qui produit lui aussi un pivotement symétrique autour de l'axe que constitue dans cette ocuvre la note Do (Charru op.cit.). Cettc modification du point d'ancrage de la mélodie contribue certes à configurer une variation du thème. mais ceci à un niveau hićrarchique subséquent à la mutation des registres. 
33 SCHOENBERG, A., 1967 : Fundamentals of musical composition. Faber \& Faber, New York.

34. Cette section se caractérise aussi par la haute inharmonicité de ses intervalles verticaux. fait très rare dans cette ocuvre baséc sur des intervalles consonants L'harmonicité relative des objets sonores fait l'objet d'un autre module algorithmique dans le même cnvironnement informatique

35. La courbe des amplitudes reproduit la nuance moyenne observéc dans chacun des $1 \pm$ objets qui segmentent la section. Ces nuances accomplissent la sćquence suivante : pp - crescendo - p - crescendo - $f$ - crescendo - ff molto diminuendo - $\mathrm{p}$ - diminuendo. Le coefficient de corrélation entre les deux courbes est nettement positif : 0.55

36. JANKÉLÉVITCH. V., 1968 (op.cit.), HALBREICH. H.. 1980 : “Analyse de l'ocuvre" |in LOCKSPEISER op.cit.|. ct al..

37. Rappelons que le laux d'occupation de l'espace (AMBITUS) est ici de 0.91, et que le taux d'utilisation des 7 registres est à son maximum. 1.

38. BARRAQUE, J. 1988 : La.Mer. Analyse Musicale nं 12 (texte posthume).

39. Becthoven avait déjà pleinement conscience du potentiel de la structuration des sonorités pures pour l'expression de la forme : voir GUIGUE. D.. 1994: "Beethoven et le pianoforte : l'émergence d'une penséc des timbres comme dimension autonome du discours musical", Revte de . fusicologie $n^{\circ} 8() / I$.

Didier Guigue é musicólogo e professor da Universidade Federal da Paraíba. 